\title{
Rare MLL-ELL fusion transcripts in childhood acute myeloid leukemia- association with young age and myeloid sarcomas?
}

\author{
Ioannis Panagopoulos ${ }^{1,2^{*}}$, Ludmila Gorunova ${ }^{1,2}$, Gitte Kerndrup ${ }^{3}$, Signe Spetalen ${ }^{4}$, Anne Tierens ${ }^{5}$, \\ Liv T. N. Osnes ${ }^{6}$, Kristin Andersen ${ }^{1,2}$, Lil-Sofie Ording Müller ${ }^{7}$, Marit Hellebostad ${ }^{8}$, Bernward Zeller ${ }^{9}$ \\ and Sverre Heim ${ }^{1,2,10}$
}

\begin{abstract}
Background: The chromosomal translocation $\mathrm{t}(11 ; 19)(\mathrm{q} 23 ; \mathrm{p} 13)$ with a breakpoint within subband 19p13.1 is found mainly in acute myeloid leukemia (AML) and results in the MLL-ELL fusion gene. Variations in the structure of MLL-ELL seem to influence the leukemogenic potency of the fusion in vivo and may lie behind differences in clinical features. The number of cases reported so far is very limited and the addition of more information about MLL-ELL variants is essential if the possible clinical significance of rare fusions is to be determined.

Case presentation: Cytogenetic and molecular genetic analyses were done on the bone marrow cells of a 20-month-old boy with an unusual form of myelomonocytic AML with multiple myeloid sarcomas infiltrating bone and soft tissues. The G-banding analysis together with FISH yielded the karyotype 47,XY, +6,t(8;19;11)(q24;p13;q23). FISH analysis also demonstrated that MLL was split. RNA-sequencing showed that the translocation had generated an MLL-ELL chimera in which exon 9 of $M L L$ (nt 4241 in sequence with accession number NM_005933.3) was fused to exon 6 of ELL (nt 817 in sequence with accession number NM_006532.3). RT-PCR together with Sanger sequencing verified the presence of the above-mentioned fusion transcript.
\end{abstract}

Conclusions: Based on our findings and information on a few previously reported patients, we speculate that young age, myelomonoblastic AML, and the presence of extramedullary disease may be typical of children with rare MLL-ELL fusion transcripts.

Keywords: Acute myeloid leukemia, Myeloid sarcoma, RNA-sequencing, Chromosomal translocation, $\mathrm{t}(11 ; 19)(q 23 ; p 13), M L L, E L L$, Fusion gene

\section{Background}

The chromosomal translocation $\mathrm{t}(11 ; 19)(\mathrm{q} 23 ; \mathrm{p} 13)$ has been reported in both acute myeloid (AML) and acute lymphoblastic leukemia (ALL) [1]. Breakpoints within subband 19p13.3 are found in both ALL (primarily in infants and children) and AML with the translocation

\footnotetext{
${ }^{*}$ Correspondence: ioannis.panagopoulos@rr-research.no

1 Section for Cancer Cytogenetics, Institute for Cancer Genetics and Informatics, The Norwegian Radium Hospital, Oslo University Hospital, Nydalen, P.O.Box 4953, 0424 Oslo, Norway

Full list of author information is available at the end of the article
}

$\mathrm{t}(11 ; 19)$ (q23;p13.3) leading to the fusion of $M L L$ with MLLT1 (also known as ENL, LTG19, and YEATS1) generating an $M L L-M L L T 1$ fusion gene [2]. Breakpoints within subband $19 \mathrm{p} 13.1$ are found mostly in AML where the translocation $\mathrm{t}(11 ; 19)(\mathrm{q} 23 ; \mathrm{p} 13.1)$ results in the $M L L-E L L$ fusion gene [3]. $M L L-E L L$ fusions were recently found also in two biphenotypic leukemias [4]. Two other $M L L-$ fusion genes have also been reported in $\mathrm{t}(11 ; 19)$-positive AML. A recurrent $M L L-M Y O 1 F$ [translocation $\mathrm{t}(11 ; 19)$ (q23;p13.2)] fusion gene was seen in infant AML $[5,6]$, whereas an MLL-SH3GL1 fusion [translocation $\mathrm{t}(11 ; 19)$ 
(q23;p13.3)] was reported in a case of childhood AML [7].

In the majority of $M L L-E L L$ fusion transcripts, exon 9, 10,11 or 12 of $M L L$ is fused to exon 2 of $E L L$ [3, 8-13]. A variant form of $M L L-E L L$ fusion transcript has been reported in chronic myelomonocytic leukemia in which $M L L$ exon 9 (exon 10 according to Nilson et al. [14]) was fused to ELL exon 3 [15]. Furthermore, in a case of congenital acute monoblastic leukemia with a three-way translocation $t(1 ; 19 ; 11)(\mathrm{p} 36 ; \mathrm{p} 13.1 ; \mathrm{q} 23)$, De Braekeler et al. showed that the genomic breakpoints in $M L L$ and $E L L$ occurred in introns 9 and 5, respectively $[8,16]$.

The leukemogenic potency of $M L L-E L L$ fusion genes was demonstrated in murine model systems [17]. Moreover, variant forms of $M L L-E L L$ were shown to impair transforming activities in vitro [12]. These observations suggest that variations in $M L L-E L L$ structure may influence leukemogenic potency of the fusion also in vivo, and they hint that such variability may be behind variation in clinical features. Because so few such cases have been reported, the addition of more cases with $M L L-E L L$ variants is essential if the possible clinical significance of rarer fusions is to be determined. In the present study, we report a childhood leukemia in which a three-way translocation caused the fusion of exon 9 of $M L L$ with exon 6 of $E L L$. To the best of our knowledge, this is only the second case in which exon 6 of $E L L$ was found to be fused to $M L L[8,16,18]$.

\section{Case presentation}

\section{Ethics statement}

The study was approved by the regional ethics committee (Regional komité for medisinsk forskningsetikk Sør-Øst, Norge, http://helseforskning.etikkom.no), and written informed consent was obtained from the patient's parents to publication of the case details. The ethics committee's approval included a review of the consent procedure. All patient information has been anonymized.

\section{Clinical presentation}

A 20-month-old, previously healthy boy presented with intermittent pain in the hip. The boy's general condition was reduced, he was pale and weak, and passive movements in the right hip were painful. His spleen was slightly enlarged, whereas liver size was normal. Blood tests revealed anemia (hemoglobin $8.2 \mathrm{~g} / \mathrm{dL}$ ), thrombocytopenia $\left(64 \times 10^{9} / \mathrm{L}\right)$, normal white blood cell count $\left(11.9 \times 10^{9} / \mathrm{L}\right)$ with monocytosis $\left(1.5 \times 10^{9} / \mathrm{L}\right)$, and immature myeloid cells were seen in the blood smear (Fig. 1).

A bone marrow investigation (which was repeated several times due to diagnostic difficulties) and a biopsy from one of the bony lesions revealed morphologically a

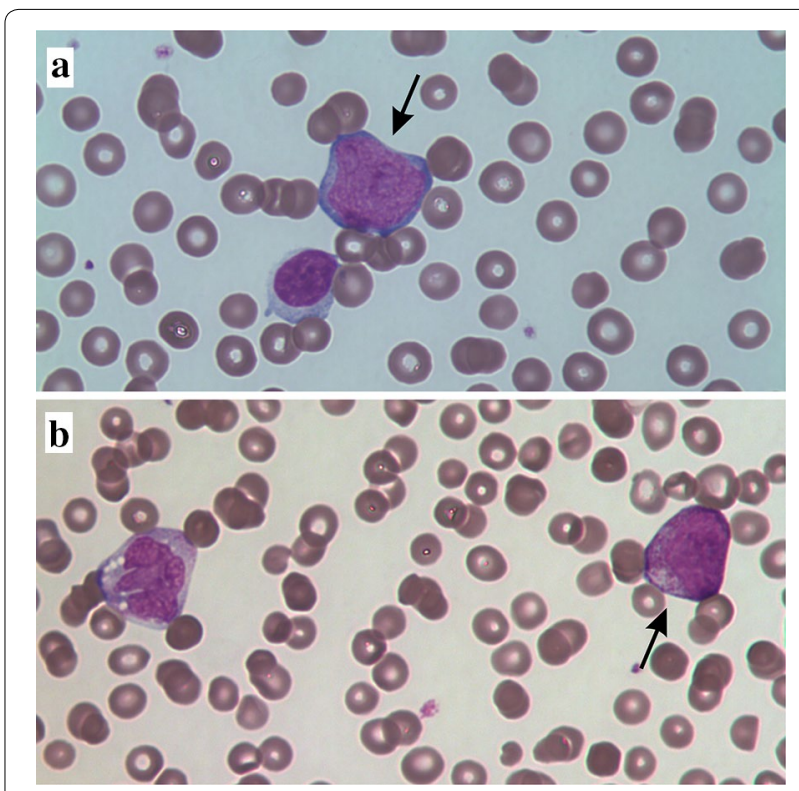

Fig. 1 Microscopic picture of peripheral blood smear of the AML patient from the time of admission to the local pediatric department showing a a myeloblast (arrow) and a lymphocyte. b A promyelocyte (arrow) and a vacuolated monocyte. Magnification 100x

myeloid hyperplasia comprised of predominantly monomorphonuclear cells. Erythropoiesis and megakaryopoiesis were sparse without dysplasia and decreased further with time until they were nearly absent in the later biopsies. The monomorphonuclear cells were positive for CD4 and CD56. The proportion of CD34+ and CD117+ cells was not increased. Further, flow cytometry analysis (Fig. 2) confirmed the normal distribution of $\mathrm{CD} 34^{+}$or $\mathrm{CD} 117^{+}$precursor cells but revealed two distinct myeloid populations comprised of $68 \% \mathrm{CD} 15$ bright $+/ \mathrm{HLA}^{-D R^{-}}$ granulocytic cells and $15 \%$ CD64 bright $+/$ HLA-DR $^{-}$ monocytic cells. Both lineages showed marker expression consistent with a shift to early stages as well as abnormal expression of CD56. In addition, CD13, a common myeloid marker, was not expressed in the early stages of these myeloid populations whereas abnormal expression of CD4 was demonstrated on early granulocytes. Only $50 \%$ of the granulocytes had acquired expression of CD11b and to a lesser extent CD16 which is consistent with maturation beyond the promyelocyte stage. However, $\mathrm{CD} 10^{+} / \mathrm{CD} 16^{+}$end-stage neutrophils were not identified. Around $20 \%$ of the monocytes were negative for CD35 and CD14 which are features of promonocytes. Based on the above immunophenotypic findings alone, a definitive diagnosis of acute myeloid leukemia could not be made.

Whole body magnetic resonance imaging (WBMRI) showed marked spiculated periosteal reaction and new 


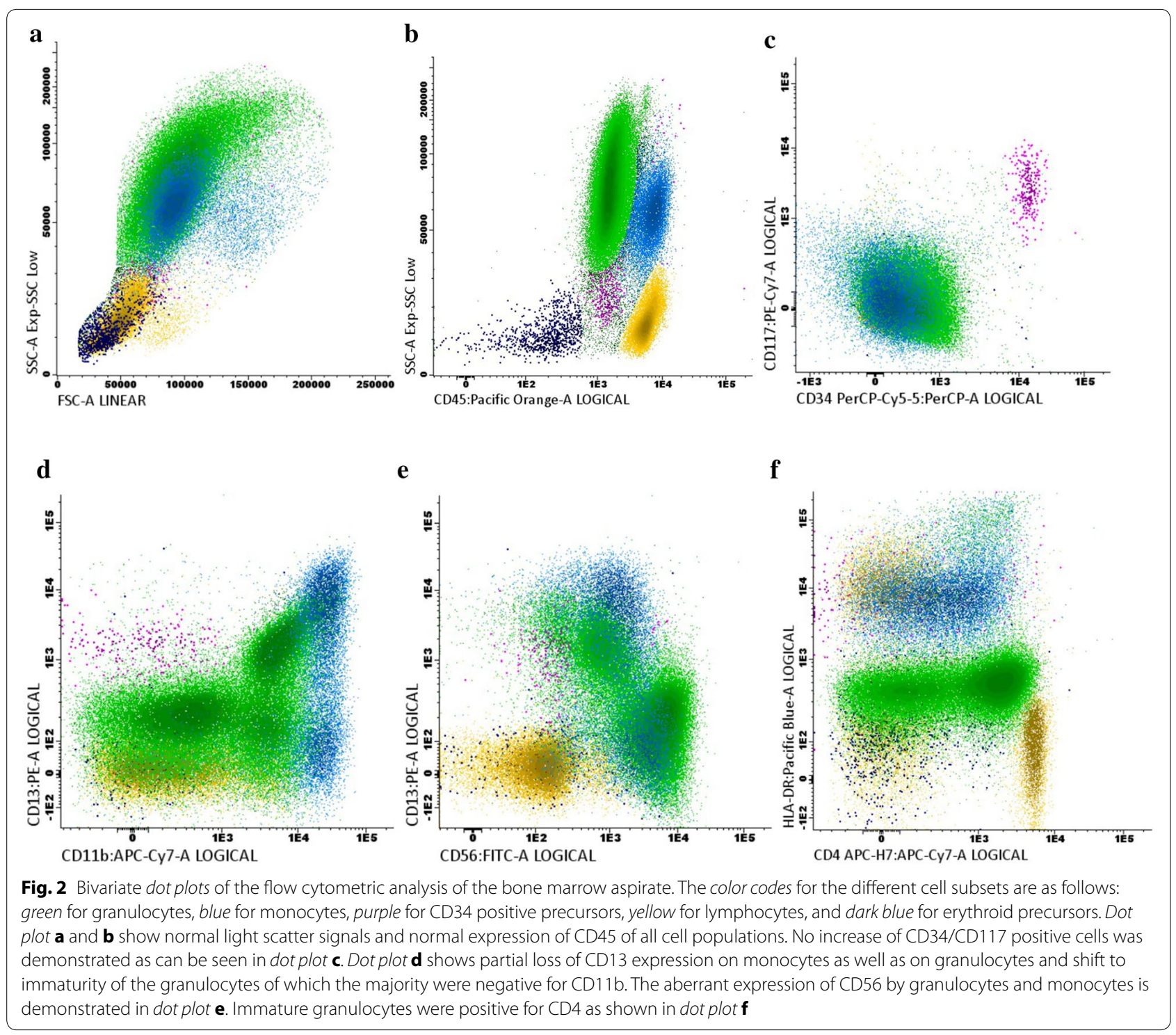

bone formation in the mandible, iliac bones, and bilaterally in the distal femur (Fig. 3a-d). In addition, there were widespread bone lesions with infarctions and necroses involving the skull base, mandible, scapulae, pelvic bones, femurs, and tibia. There was soft tissue involvement of the lower extremities with edema in the muscles and subcutaneous tissue and fluid along the facial planes (Fig. 3a-d).

The G-banding analysis of bone marrow metaphase cells at diagnosis showed 11 cells carrying an extra chromosome 6 as well as the chromosomal translocation $\mathrm{t}(11 ; 19)(\mathrm{q} 23 ; \mathrm{p} 13)$ yielding the karyotype 47,XY, $+6, \mathrm{t}(11 ; 19)$ (q23;p13) [11] (Fig. 4a).

Interphase FISH analyses of bone marrow cells using the Cytocell multiprobe ALL panel (Cytocell, http://www. cytocell.co.uk/) showed a split signal of the $M L L$ locus in 146 out of 201 investigated interphase nuclei (data not shown). FISH analysis of metaphase spreads using the $M L L$ breakpoint probe (Cytocell, http://www.cytocell. co.uk/) showed that the red signal (distal) had moved not to chromosome 19 but to the $\mathrm{q}$ arm of chromosome 8 (Fig. 4b). Thus, the modified karyotype after G-banding analysis and FISH was 47,XY, +6,t(8;19;11)(q24;p13;q23) [11] (Fig. 4a, b).

Mainly on the basis of the detected $M L L$-rearrangement, we interpreted the boy's disease as an unusual form of myelomonocytic AML with multiple myeloid sarcomas infiltrating bone and soft tissues.

During the investigation, the patient's extensive skeletal lesions increased causing therapy-resistant pain and 


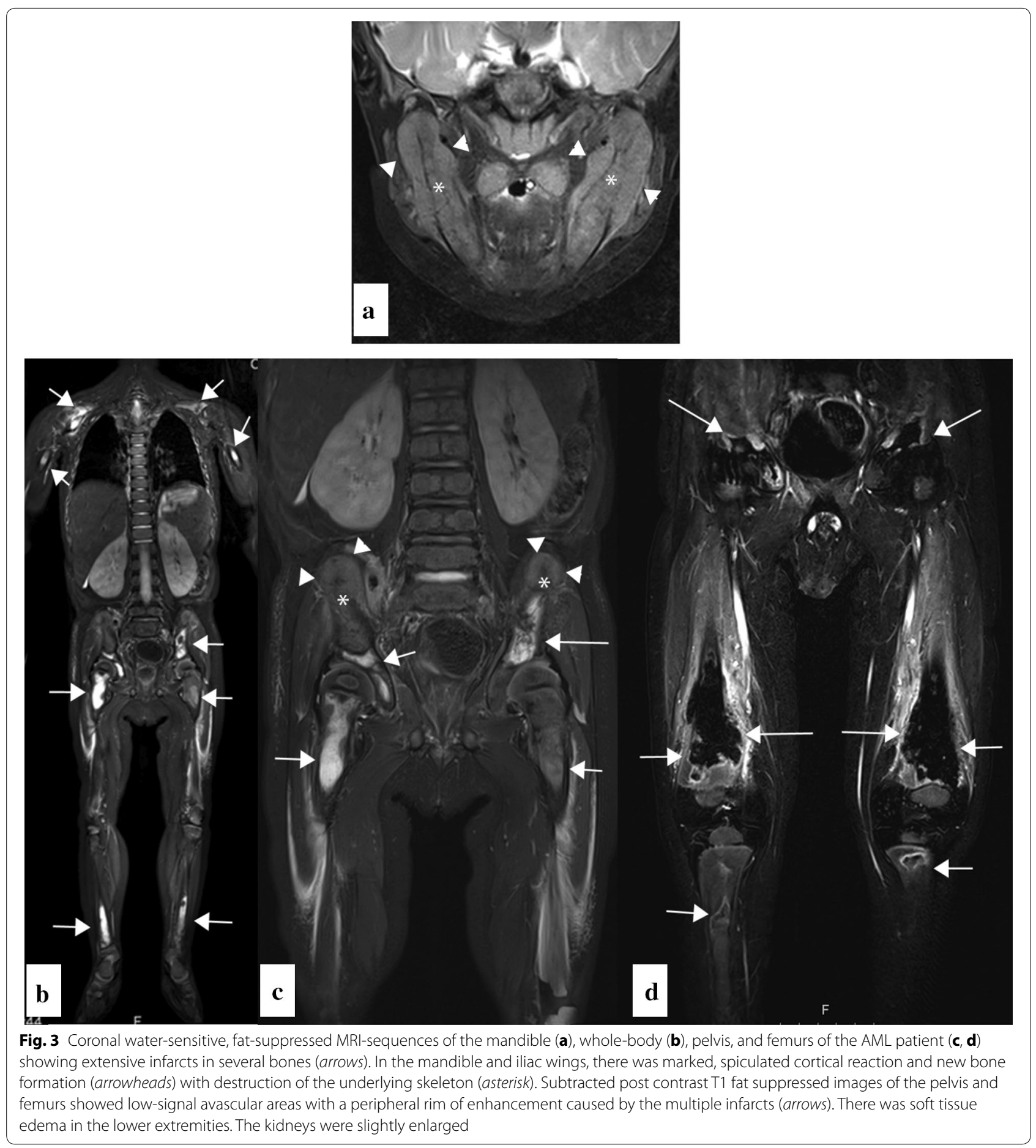

his general condition deteriorated. After 3 weeks, AMLdirected therapy was begun according to the NOPHODBH AML 2012 protocol [19]. He received five courses (MEC, ADxE, HAM, HA3E, FLA) at 5-6 weeks intervals. For details, see Additional file 1: Figure: S1.
Clinically, the boy recovered rather quickly. Evaluation before course two showed no remaining $M L L$-rearranged cells by FISH in the bone marrow. MRI controls of his bony lesions demonstrated continuous, but slow, regression. At 9 months after cessation of treatment, the boy is 
$\mathbf{a}$

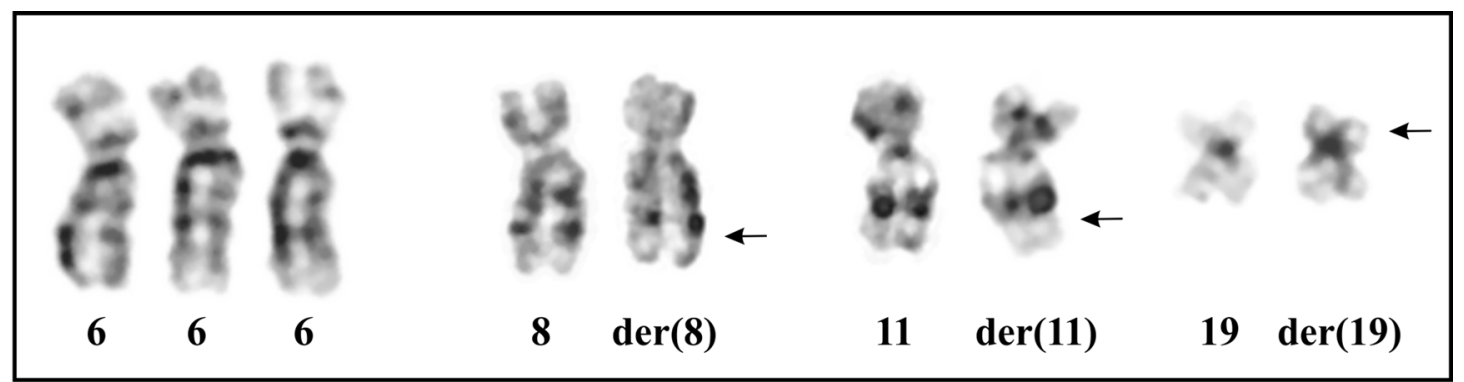

b

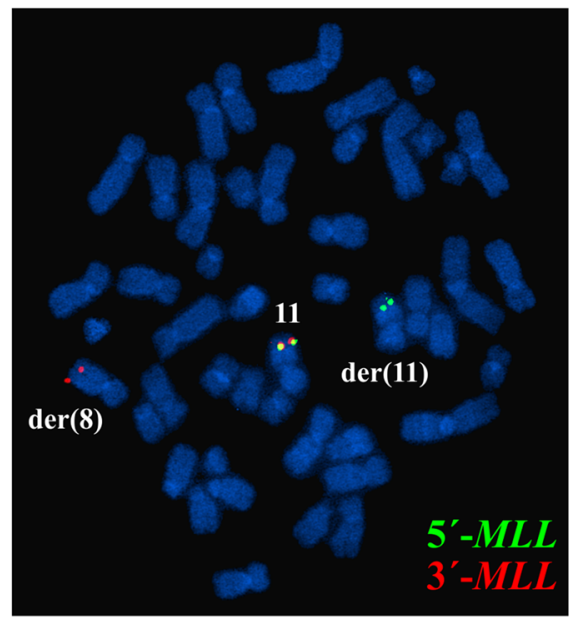

c

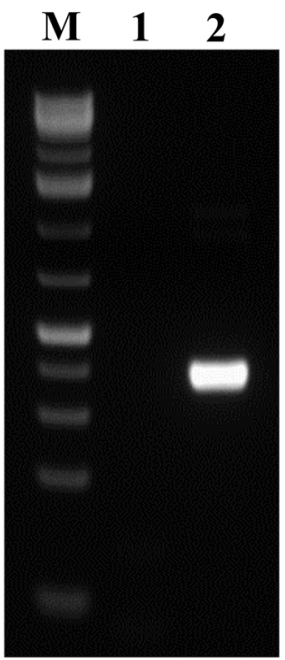

d

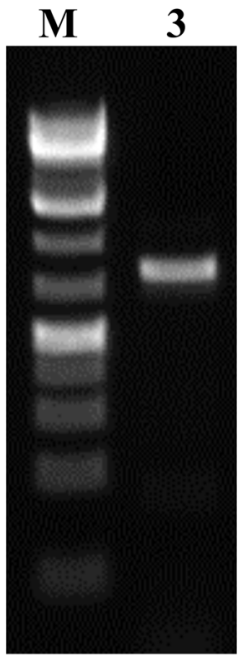

\section{e}

\section{MLL exon $9 \downarrow E L L$ exon 6}

\section{A GA GTGGA CTTTAA GGTGGC CAA CA TGA GT}

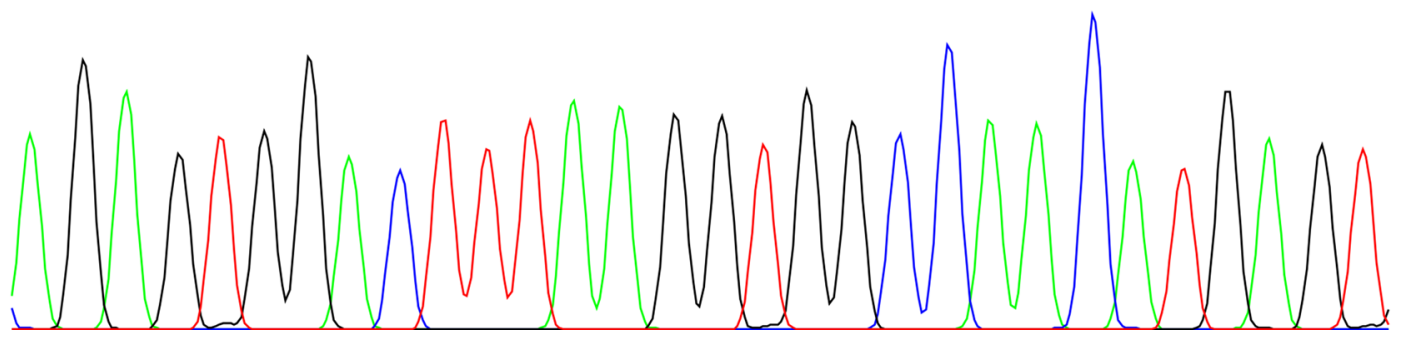

Fig. 4 Cytogenetic, FISH, and PCR analyses of the AML patient. a G-banded karyotype showing trisomy 6, der(8), der(11), and der(19) of the $\mathrm{t}(8 ; 19 ; 11)(\mathrm{q} 24 ; \mathrm{p} 13 ; \mathrm{q} 23)$ together with the corresponding normal chromosome homologs; breakpoint positions are indicated by arrows. b FISH using an $M L L$ breakapart probe showed rearrangement of $M L L$. The $3^{\prime}$-end part of the MLL gene (red probe) has moved to the $q$ arm of the der(8), while the $5^{\prime}$-end part of the gene (green probe) remains on 11q23 of the der(11). c The initial RT-PCR amplifications for the detection of a possible MLL-ELL fusion transcript. Lane 1, nested PCR with the forward primers located in exon 7 of MLL and reverse primers located in exon 4 of ELL (MLL3947F1/ELL-415R) failed to amplify any CDNA fragments. Lane 2, amplification of a cDNA fragment of the ABL1 gene using the primers ABL1-91F1 and AsBL1-404R1 suggested that the synthesized CDNA was of good quality. d RT-PCR using a new reverse primer located in exon 8 of ELL (primer ELL-1044R1) and a forward primer located in exon 7 of MLL (primer MLL-3878F) amplified a cDNA fragment. M, 1 Kb DNA ladder. e Partial sequence chromatogram of the amplified fragment using the primers MLL-3878F and ELL-1044R1 showing the junction of the MLL-ELL chimeric transcript 
clinically healthy. As expected, MRI still shows several small residual bone lesions undergoing regression. Unexpectedly, however, the patient has developed a new, small (about $5 \%$ ) clone in the bone marrow with a solitary $7 q$ deletion. This may or may not represent an emerging secondary malignancy [20-23], and the situation is being monitored closely to see whether the clone expands and gives rise to hematologically recognizable disease.

\section{Initial RT-PCR experiments}

Total RNA, isolated from the patient's bone marrow at the time of diagnosis, was reverse-transcribed using iScript Advanced cDNA Synthesis Kit for RT-qPCR (BioRad Laboratories, Oslo, Norway) and cDNA corresponding to $20 \mathrm{ng}$ total RNA was used as template in PCR amplifications as previously described $[24,25]$. The initial RT-PCR with the primer set MLL-3878F/ELL-498R1 as well as nested PCR with the primers MLL-3947F1/ELL415R (the sequences of the primers are listed in Table 1) failed to amplify any cDNA fragments (Fig. 4c). At the same time, use of the primer set ABL1-91F/ABL1-404R1 led to amplification of an $A B L 1$ cDNA fragment suggesting that the synthesized cDNA was of good quality (Fig. 4c).

\section{RNA-sequencing}

Because of the negative RT-PCR results, the less than typical cytogenetic findings, and the clinical picture, three $\mu \mathrm{g}$ of the total RNA extracted from the patients' bone marrow at the time of diagnosis were subjected to high-throughput paired-end RNA-sequencing at the Norwegian Sequencing Centre, Oslo University Hospital (http://www.sequencing.uio.no/) as described elsewhere $[24,26]$. The raw sequencing data were subsequently analyzed using FusionCatcher which is a program designed to detect fusion genes from high throughput sequencing data [27]. More than 100 potential fusion transcripts were found (Additional file 2: Table: S1), among them an
$M L L-E L L$ in which exon 9 of $M L L$ (nt 4241 in sequence with accession number NM_005933.3) was fused to exon 6 of $E L L$ (nt 817 in sequence with accession number NM_006532.3). No reciprocal ELL-MLL fusion transcript was found.

In order to verify the fusion obtained with FusionCatcher, we used the "grep" command utility [28] to search for expressions composed of $10 \mathrm{nt}$ of $M L L$ and 10 nt of ELL upstream and downstream of the fusion point, respectively (Table 2 ). Using the expression "GACTT TAAGGTGGCCAACAT" which is composed of 10 nt, "GACTTTAAGG", from $M L L$ and $10 \mathrm{nt}$, "TGGC CAACAT", from $E L L, 38$ sequences were retrieved (Table 2).

\section{Molecular genetic confirmation of the fusion}

PCR with the MLL-3878F and ELL-1044R1 primer combination (Table 1) amplified a fragment from the patient's bone marrow cDNA (Fig. 4d). Sanger sequencing of the amplified product showed that it was a chimeric $M L L-$ $E L L$ cDNA fragment in which exon 9 of $M L L$ was fused to exon 6 of $E L L$, i.e., the same $M L L-E L L$ fusion transcript found by RNA-sequencing (Fig. 4e; Table 2).

\section{Conclusions}

We report a case of AML genetically characterized by a three-way translocation, $\mathrm{t}(8 ; 19 ; 11)(\mathrm{q} 24 ; \mathrm{p} 13 ; \mathrm{q} 23)$, leading to rearrangement of the $M L L$ gene and the generation of a chimeric $M L L-E L L$ transcript with fusion of $M L L$ exon 9 to $E L L$ exon 6. The initial RT-PCR amplifications relied on forward primers located in exon 7 of $M L L$ and reverse primers located in exon 4 of $E L L$; this choice was based on findings in previous studies in which $M L L$ was shown to fuse with exon 2 or 3 of $E L L[3,10,11,15,29,30]$. The PCRs with these primer sets (first PCR with MLL-3878F/ ELL-498R1, then nested PCR with the primers MLL3947F1/ELL-415R) failed to amplify any cDNA fragments. It was a combination of three methods-banding

Table 1 Primers used for PCR amplification and Sanger sequencing analyses

\begin{tabular}{|c|c|c|c|c|c|}
\hline Name & Sequence $\left(5^{\prime}->3^{\prime}\right)$ & Direction & Position/exon & Reference sequence & Gene \\
\hline MLL-3735F & CCCATCAGCAAGAGAGGATCCTGC & Forward & $3758-3781 / 7$ & NM_005933.3 & $M L L(K M T 2 A)$ \\
\hline MLL-3878F & AGTCAAGCAAGCAGGTCTCCCAGC & Forward & $3901-3924 / 7$ & NM_005933.3 & $M L L(K M T 2 A)$ \\
\hline MLL-3947F1 & GCCACCTACTACAGGACCGCCAAG & Forward & $3947-3970 / 7$ & NM_005933.3 & $M L L(K M T 2 A)$ \\
\hline ELL-415R1 & GGCACACACCGTGATCTTGTCCTG & Reverse & $438-415 / 4$ & NM_006532.3 & $E L L$ \\
\hline ELL-498R1 & TTGATGACAATGGCACTTCGGCTC & Reverse & $498-521 / 4$ & NM_006532.3 & $E L L$ \\
\hline ELL-960R1 & TCCAAGGAGGCTGCCAGTGCTC & Reverse & $981-960 / 7$ & NM_006532.3 & $E L L$ \\
\hline ELL-1044R1 & CGATGAAATCAGGAGGCTGCAGC & Reverse & $1066-1044 / 8$ & NM_006532.3 & $E L L$ \\
\hline$A B L 1-91 F 1$ & CAGCGGCCAGTAGCATCTGACTTTG & Forward & $280-304 / 2$ & NM_005157.5 & $A B L 1$ \\
\hline ABL 1-404R1 & CTCAGCAGATACTCAGCGGCATTGC & Reverse & $617-593 / 3$ & NM_005157.5 & $A B L 1$ \\
\hline
\end{tabular}


Table 2 Sequences retrieved with the "grep» command using the expression "GACTTTAAGGTGGCCAACAT"

ATGGAGTCCACAGGATCAGAGTGGACTTTAAGGTGGCCAACATGAGTGCTAAGGACGGCACGTGTACACTGCAGGACTGCATGTACAAGGATGTGCAGAAGGACTGGCCTGGCTACTCGGAGGGGG

GTTCTAAGCAAAAAATTCCAGCAGATGGAGTCCACAGGATCAGAGTGGACTTTAAGGTGGCCAACATGAGTGCTAAGGACGGCACGTGTACACTGCAGGACTGCATGTACAAGGATGTGCAGAAGG

AAAAAATTCCAGCAGATGGAGTCCACAGGATCAGAGTGGACTTTAAGGTGGCCAACATGAGTGCTAAGGACGGCACGTGTACACTGCAGGACTGCATGTACAAGGATGTGCAGAAGGACTGGCCTG

CCTCAGCACTCTCTCCAATGGCAATAGTTCTAAGCAAAAAATTCCAGCAGATGGAGTCCACAGGATCAGAGTGGACTTTAAGGTGGCCAACATGAGTGCTAAGGACGGCACGTGTACACTGCAGGA

GCACTCTCTCCAATGGCAATAGTTCTAAGCAAAAAATTCCAGCAGATGGAGTCCACAGGATCAGAGTGGACTTTAAGGTGGCCAACATGAGTGCTAAGGACGGCACGTGTACACTGCAGGACTGCA

AATGCAGGCACTTTGAACATCCTCAGCACTCTCTCCAATGGCAATAGTTCTAAGCAAAAAATTCCAGCAGATGGAGTCCACAGGATCAGAGTGGACTTTAAGGTGGCCAACATGAGTGCTAAGGAC

GTGGACTTTAAGGTGGCCAACATGAGTGCTAAGGACGGCACGTGTACACTGCAGGACTGCATGTACAAGGATGTGCAGAAGGACTGGCCTGGCTACTCGGAGGGGGACCAGCAGCTGCTGAAGCGG

CCTCAGCACTCTCTCCAATGGCAATAGTTCTAAGCAAAAAATTCCAGCAGATGGAGTCCACAGGATCAGAGTGGACTTTAAGGTGGCCAACATGAGTGCTAAGGACGGCACGTGTACACTGCAGGA

CCTCAGCACTCTCTCCAATGGCAATAGTTCTAAGCAAAAAATTCCAGCAGATGGAGTCCACAGGATCAGAGTGGACTTTAAGGTGGCCAACATGAGTGCTAAGGACGGCACGTGTACACTGCAGGA

CTCTCTCCAATGGCAATAGTTCTAAGCAAAAAATTCCAGCAGATGGAGTCCACAGGATCAGAGTGGACTTTAAGGTGGCCAACATGAGTGCTAAGGACGGCACGTGTACACTGCAGGACTGCATGT

CTCTCTCCAATGGCAATAGTTCTAAGCAAAAAATTCCAGCAGATGGAGTCCACAGGATCAGAGTGGACTTTAAGGTGGCCAACATGAGTGCTAAGGACGGCACGTGTACACTGCAGGACTGCATGT

GAACATCCTCAGCACTCTCTCCAATGGCAATAGTTCTAAGCAAAAAATTCCAGCAGATGGAGTCCACAGGATCAGAGTGGACTTTAAGGTGGCCAACATGAGTGCTAAGGACGGCAAGTGTACACT

TGAACATCCTCAGCACTCTCTCCAATGGCAATAGTTCTAAGCAAAAAATTCCAGCAGATGGAGTCCACAGGATCAGAGTGGACTTTAAGGTGGCCAACATGAGTGCTAAGGACGGCACGTGTACAC

TCTCCAATGGCAATAGTTCTAAGCAAAAAATTCCAGCAGATGGAGTCCACAGGATCAGAGTGGACTTTAAGGTGGCCAACATGAGTGCTAAGGACGGCACGTGTACACTGCAGGACTGCATGTACA

TGGACTTTAAGGTGGCCAACATGAGTGCTAAGGACGGCACGTGTACACTGCAGGACTGCATGTACAAGGATGTGCAGAAGGACTGGCCTGGCTACTCGGAGGGGGACCAGCAGCAGATCGGAAGAG

TGGACTTTAAGGTGGCCAACATGAGTGCTAAGGACGGCACGTGTACACTGCAGGACTGCATGTACAAGGATGTGCAGAAGGACTGGCCTGGCTACTCGGAGGGGGACCAGCAGCAGATCGGAAGAG

CTTTGAACATCCTCAGCACTCTCTCCAATGGCAATAGTTCTAAGCAAAAAATTCCAGCAGATGGAGTCCACAGGATCAGAGTGGACTTTAAGGTGGCCAACATGAGTGCTAAGGACGGCACGTGTA

CAGGATCAGAGTGGACTTTAAGGTGGCCAACATGAGTGCTAAGGACGGCACGTGTACACTGCAGGACTGCATGTACAAGGATGTGCAGAAGGACTGGCCTGGCTACTCGGAGGGGGACCAGCAGCT

ATCAGAGTGGACTTTAAGGTGGCCAACATGAGTGCTAAGGACGGCACGTGTACACTGCAGGACTGCATGTACAAGGATGTGCAGAAGGACTGGCCTGGCTACTCGGAGGGGGACCAGCAGCTGCTG

GGAGTCCACAGGATCAGAGTGGACTTTAAGGTGGCCAACATGAGTGCTAAGGACGGCACGTGTACACTGCAGGACTGCATGTACAAGGATGTGCAGAAGGACTGGCCTGGCTACTCGGAGGGGGAC

GCACTCTCTCCAATGGCAATAGTTCTAAGCAAAAAATTCCAGCAGATGGAGTCCACAGGATCAGAGTGGACTTTAAGGTGGCCAACATGAGTGCTAAGGACGGCACGTGTACACTGCAGGACTGCA

GCAATAGTTCTAAGCAAAAAATTCCAGCAGATGGAGTCCACAGGATCAGAGTGGACTTTAAGGTGGCCAACATGAGTGCTAAGGACGGCACGTGTACACTGCAGGACTGCATGTACAAGGATGTGC

GTGGACTTTAAGGTGGCCAACATGAGTGCTAAGGACGGCACGTGTACACTGCAGGACTGCATGTACAAGGATGTGCAGAAGGACTGGCCTGGCTACTCGGAGGGGGACCAGCAGCTGCTGAAGCGG

ATGCAGGCACTTTGAACATCCTCAGCACTCTCTCCAATGGCAATAGTTCTAAGCAAAAAATTCCAGCAGATGGAGTCCACAGGATCAGAGTGGACTTTAAGGTGGCCAACATGAGTGCTAAGGACG

CACAGGATCAGAGTGGACTTTAAGGTGGCCAACATGAGTGCTAAGGACGGCACGTGTACACTGCAGGACTGCATGTACAAGGATGTGCAGAAGGACTGGCCTGGCTACTCGGAGGGGGACAGATCG

CTCCAATGGCAATAGTTCTAAGCAAAAAATTCCAGCAGATGGAGTCCACAGGATCAGAGTGGACTTTAAGGTGGCCAACATGAGTGCTAAGGACGGCACGTGTACACTGCAGGACTGCATGTACAA

CTCCAATGGCAATAGTTCTAAGCAAAAAATTCCAGCAGATGGAGTCCACAGGATCAGAGTGGACTTTAAGGTGGCCAACATGAGTGCTAAGGACGGCACGTGTACACTGCAGGACTGCATGTACAA

CAGGCACTTTGAACATCCTCAGCACTCTCTCCAATGGCAATAGTTCTAAGCAAAAAATTCCAGCAGATGGAGTCCACAGGATCAGAGTGGACTTTAAGGTGGCCAACATGAGTGCTAAGGACGGCA

CAGCACTCTCTCCAATGGCAATAGTTCTAAGCAAAAAATTCCAGCAGATGGAGTCCACAGGATCAGAGTGGACTTTAAGGTGGCCAACATGAGTGCTAAGGACGGCACGTGTACACTGCAGGACTG 


\section{Table 2 continued}

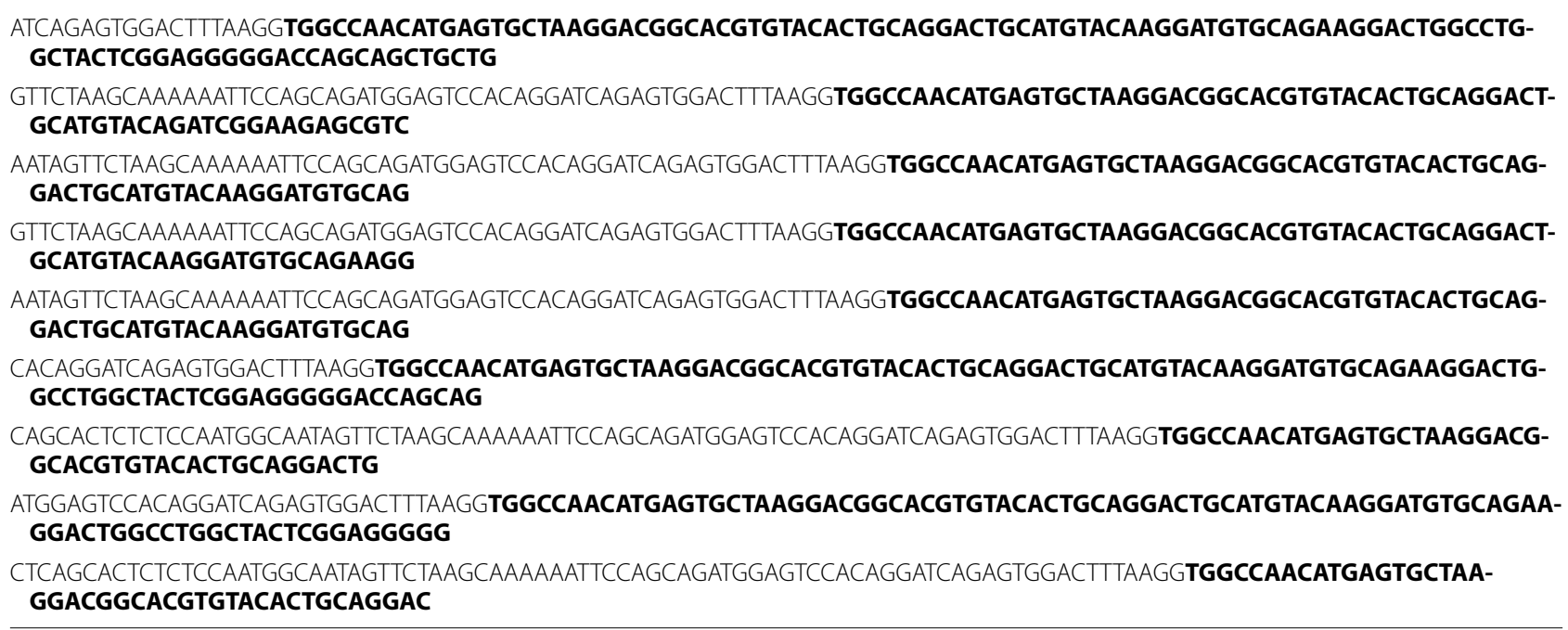

The sequences of $E L L$ are in bold

cytogenetics, FISH, and RNA-sequencing-that helped us identify the present $M L L$ exon 9-ELL exon 6 fusion. G-banding analysis showed what appeared to be a regular chromosomal translocation $\mathrm{t}(11 ; 19)(\mathrm{q} 23 ; \mathrm{p} 13)$ (Fig. 4a), a well-known change in acute leukemia. FISH showed that although $M L L$ was split, the distal part of the gene was moved not to the derivative 19 but, surprisingly, to the long arm of chromosome 8 (Fig. 4b). Finally, RNAsequencing showed that exon 9 of $M L L$ was fused to exon 6 of ELL (Table 2). RT-PCR using a new reverse primer located in exon 8 of ELL (primer ELL-1044R1, MLL$3878 \mathrm{~F}$ and ELL-1044R1 primer combination) then confirmed the fusion transcript (Fig. 4d, e).

In 2009, De Braekeleer and coworkers reported a case of congenital acute monoblastic leukemia with a threeway translocation, $\mathrm{t}(1 ; 19 ; 11)(\mathrm{p} 36 ; \mathrm{p} 13.11 ; \mathrm{q} 23)$, which involved the $M L L$ gene and generated an $M L L-E L L$ fusion identical to that of the present case $[8,16,18]$. To the best of our knowledge, these are the only two cases hitherto reported in which exon 6 of $E L L$ is fused to $M L L$. It is certainly intriguing that three-way translocations, an unusual phenomenon behind $M L L$-rearrangements, had occurred in both cases; it may hint at some currently hidden mechanism behind the generation of the genomic change. The number of AML cases with $M L L$ exon 9-ELL exon 6 fusions might actually be underestimated when assessed by means of RT-PCR amplifications using primer sets based on hitherto published studies [3, $10,11,15,29,30]$. This situation may be remedied by use of a new RT-PCR method that includes primers to detect fusion of $M L L$ also with exon 6 of $E L L$ [18].

Current knowledge holds that there are three types of $M L L-E L L$ fusion transcripts (Fig. 5). Type 1, found in the majority of cases, is characterized by $E L L$ exon 2 being fused to $5^{\prime}-M L L[3,8-13]$. Type 2 was so far found in only a single case of chronic myelomonocytic leukemia (CMML) that transformed to AML; it had exon 3 of $E L L$ fused to $5^{\prime}-M L L$ [15]. Type 3, of which the present case is one of two, is caused by a three-way translocation leading to the fusion of exon 6 of $E L L$ with $M L L$ (present case; $[8,16,18]$ ). All three MLL-ELL fusion proteins contain the aminoterminal region of MLL which includes the AT hooks, the methyltransferase domain, and the repression domain [31].

The case we describe presented unusual clinical features. Most conspicuous were the widespread, very painful bone lesions with soft tissue involvement which were interpreted as multiple myeloid sarcomas and extensive bone infarcts, not mere marrow infiltration. The validity of this diagnosis is supported by the gradual, protracted resolution of these lesions taking place during and after therapy. To the best of our knowledge, only four cases, including the present one, have been reported of very young AML patients displaying myelomonocytic features, myeloid sarcomas, and involvement of the $M L L-E L L$ fusion gene [8, 16, 32, 33]. The four patients have similar cytogenetic and genetic (MLL-ELL fusion) features. Three of them had a three-way translocation generating the $M L L-E L L$ fusion: the present case with $\mathrm{t}(8 ; 19 ; 11)$ (q24;p13;q23), a female newborn with $\mathrm{t}(1 ; 19 ; 11)$ (p36;p13.11;q23) [16, 32], and a three-month-old boy with $t(6 ; 19 ; 11)(p 22 ; p 13 ; q 23)$ [33]. None of the patients had the type $1 M L L-E L L$ fusion (see above). In the patient with $\mathrm{t}(1 ; 19 ; 11)(\mathrm{p} 36 ; \mathrm{p} 13.11 ; \mathrm{q} 23)$, the data suggest an $M L L$ exon 9-ELL exon 6 fusion transcript, similar to our case $[16,32]$. In a two-month-old child reported by De Braekeler et al, the genomic breakpoints in $M L L$ and 


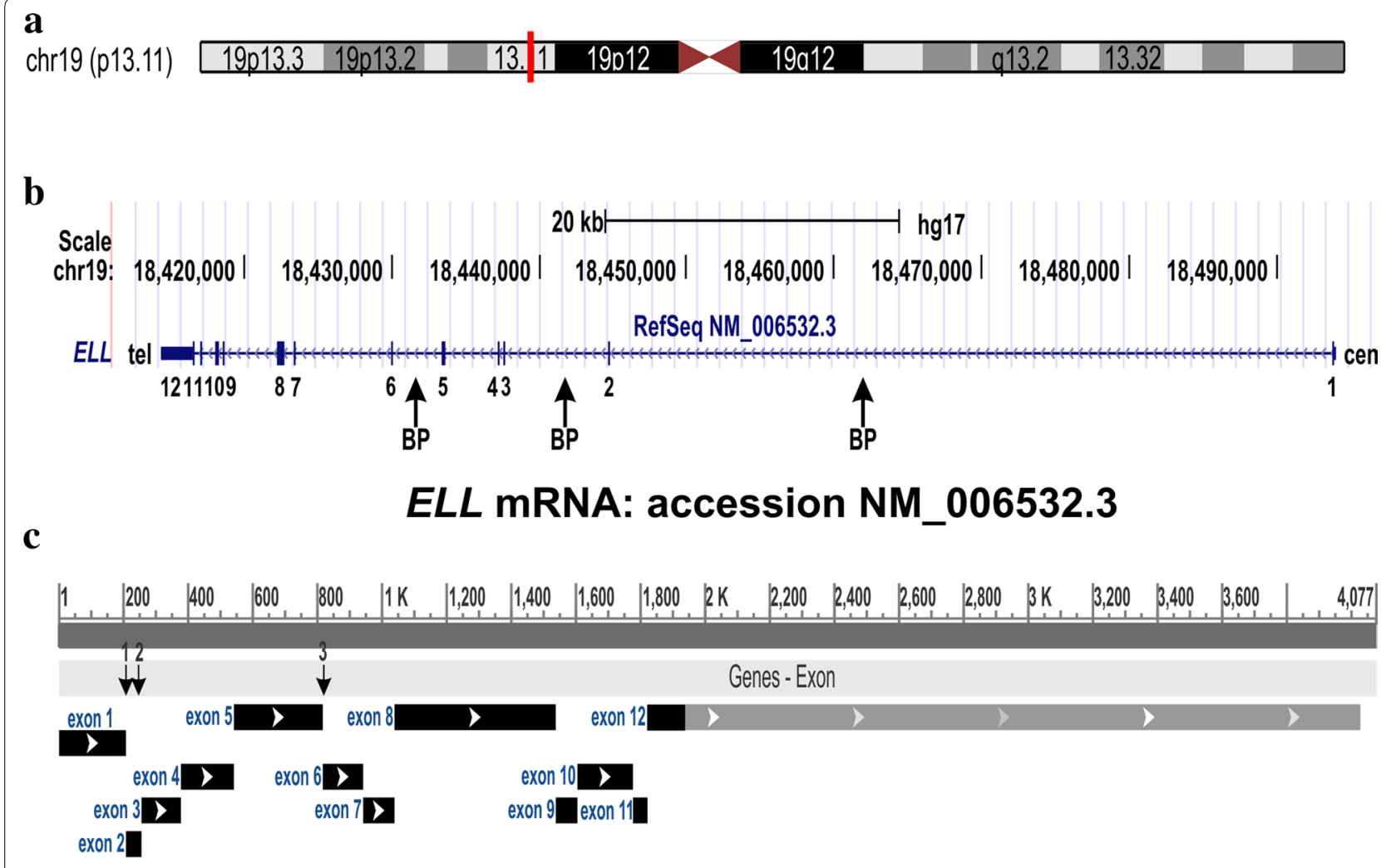

\section{d $\quad$ ELL protein: accession NP_006523.1}

Type 1

Type 2

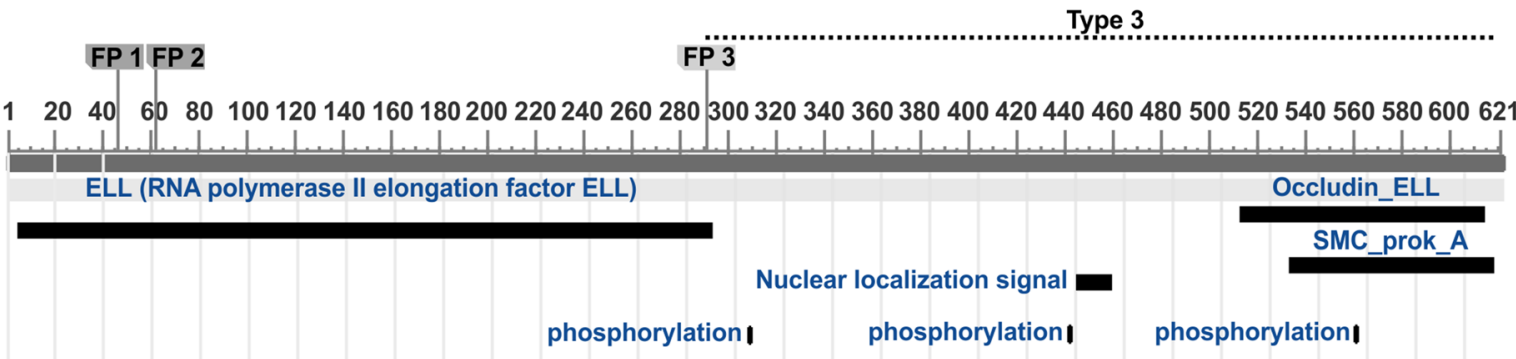

Fig. 5 Properties of the ELL gene. a Ideogram of chromosome 19 showing the location of the ELL gene in chromosome subband 19p13.11 (vertical red line). $\mathbf{b}$ Exon intron structure of the ELL gene. Vertical boxes indicate exons. The breakpoints (BP) have been found in introns 1, 2, and 5. Vertical arrows indicate the genomic BP regions. The transcription is from centromere (cen) to telomere (tel). c Diagram showing mRNA of ELL with accession number NM_006532.3. The exons which code for protein are in black. The vertical arrows indicate the fusion points 1, 2, and 3 in the MLL-ELL fusion transcripts. d Diagram showing the ELL protein, the known domains, and the phosphorylation sites. FP1, FP2, and FP3 are the fusion points with the MLL in the three types $(1,2$, and 3) of MLL-ELL fusion protein. All three MLL-ELL proteins retain the occludin homology domain, the SMC_ prok_A domain, the nuclear localization signal, and the three phosphorylation sites 
$E L L$ indicated an $M L L$ exon 9-ELL exon 3 fusion transcript $[8,32]$. In the three-month-old boy with $\mathrm{t}(6 ; 19 ; 11)$ (p22;p13;q23), the translocation resulted in an $M L L$ exon 8-ELL exon 3 fusion transcript [33].

Due to the small number of patients described, it is impossible to make definite statements about prognosis. Nevertheless, treatment results so far on patients carrying rare $M L L-E L L$ fusion genes seem to have been encouraging. The newborn patient did not receive antileukemic therapy and died $24 \mathrm{~h}$ after birth [32], but the three treated patients, including the present case, went into remission $[32,33]$. Two of them seem to be long-term survivors [32, 33], and our patient is in complete clinical remission 1 year after diagnosis. The clinical importance of the small clone with a 7q deletion that has emerged in remission is unclear. Recurrent cytogenetic abnormalities are sometimes seen in AML and ALL patients who are in complete clinical remission and may persist for years in the bone marrow even in the absence of progression to leukemia [20-23]. A wait-and-see approach is therefore prudent.

\section{Additional files}

Additional file 1: Figure S1. Treatment overview NOPHO-DBH AML2012 protocol (standard arm).

Additional file 2: Table S1. Fusion transcripts detected using FusionCatcher.

\section{Authors' contributions}

IP designed the research, performed the molecular genetic analyses, interpreted the data, and wrote the manuscript. LG and KA performed the cytogenetics and FISH experiments and interpreted the data. GK, SS, and AT did the hematopathological and immunological evaluations. LTNO made flow cytometry and immunophenotyping, evaluation of the flowcytometric data, and immunologic evaluation. L-SOM did the MRI examinations. MH treated the patient. BZ treated the patient, supervised the project, and wrote the manuscript. SH supervised the project, designed the research, evaluated the cytogenetics and FISH data, and wrote the manuscript. All authors read and approved the final manuscript.

\section{Author details}

${ }^{1}$ Section for Cancer Cytogenetics, Institute for Cancer Genetics and Informatics, The Norwegian Radium Hospital, Oslo University Hospital, Nydalen, P.O.Box 4953, 0424 Oslo, Norway. ${ }^{2}$ Centre for Cancer Biomedicine, Faculty of Medicine, University of Oslo, Oslo, Norway. ${ }^{3}$ Department of Pathology, Aarhus University Hospital, Aarhus, Denmark. ${ }^{4}$ Department of Pathology, Oslo University Hospital, Oslo, Norway. ${ }^{5}$ Laboratory Medicine Program, Department of Haematopathology, University Health Network, Toronto, Canada. ${ }^{6}$ Department of Immunology, Oslo University Hospital, Oslo, Norway. ${ }^{7}$ Department of Radiology, Oslo University Hospital, Oslo, Norway. ${ }^{8}$ Department of Pediatrics, Drammen Hospital, Vestre Viken HF, Drammen, Norway. ${ }^{9}$ Department of Pediatrics, Oslo University Hospital, Oslo, Norway. ${ }^{10}$ Faculty of Medicine, University of Oslo, Oslo, Norway.
}

\section{Acknowledgements}

This work was supported by Grants from the Norwegian Radium Hospital Foundation.

\section{Competing interests}

The authors declare that they have no competing interests.
Received: 20 November 2015 Accepted: 1 March 2016

Published online: 05 March 2016

\section{References}

1. Heim S, Mitelman F. Cancer cytogenetics: chromosomal and molecular genetic aberrations of tumor cells. 4th ed. Hoboken: Wiley-Blackwell; 2015.

2. Tkachuk DC, Kohler S, Cleary ML. Involvement of a homolog of Drosophila trithorax by 11q23 chromosomal translocations in acute leukemias. Cell. 1992;71(4):691-700.

3. Thirman MJ, Levitan DA, Kobayashi H, Simon MC, Rowley JD. Cloning of ELL, a gene that fuses to MLL in a $\mathrm{t}(11 ; 19)(\mathrm{q} 23 ; \mathrm{p} 13.1)$ in acute myeloid leukemia. Proc Natl Acad Sci USA. 1994;91(25):12110-4

4. Turkmen S, Timmermann B, Bartels G, Groger D, Meyer C, Schwartz S, et al. Involvement of the MLL gene in adult T-lymphoblastic leukemia. Genes Chromosom Cancer. 2012;51(12):1114-24. doi:10.1002/gcc.21996.

5. Duhoux FP, Ameye G, Libouton JM, Bahloula K, lossifidis S, Chantrain CF, et al. The t(11;19)(q23;p13) fusing MLL with MYO1F is recurrent in infant acute myeloid leukemias. Leuk Res. 2011;35(9):e171-2. doi:10.1016/j. leukres.2011.04.022

6. Taki T, Akiyama M, Saito S, Ono R, Taniwaki M, Kato Y, et al. The MYO1F, unconventional myosin type $1 \mathrm{~F}$, gene is fused to $\mathrm{MLL}$ in infant acute monocytic leukemia with a complex translocation involving chromosomes 7, 11, 19 and 22. Oncogene. 2005;24(33):5191-7. doi:10.1038/ sj.onc. 1208711.

7. So CW, Caldas C, Liu MM, Chen SJ, Huang QH, Gu LJ, et al. EEN encodes for a member of a new family of proteins containing an Src homology 3 domain and is the third gene located on chromosome 19p13 that fuses to MLL in human leukemia. Proc Natl Acad Sci USA. 1997;94(6):2563-8.

8. De Braekeleer E, Meyer C, Douet-Guilbert N, Basinko A, Le Bris MJ, Morel F, et al. Identification of MLL partner genes in 27 patients with acute leukemia from a single cytogenetic laboratory. Mol Oncol. 2011;5(6):555-63. doi:10.1016/j.molonc.2011.08.003.

9. Megonigal MD, Rappaport EF, Wilson RB, Jones DH, Whitlock JA, Ortega $J A$, et al. Panhandle PCR for CDNA: a rapid method for isolation of MLL fusion transcripts involving unknown partner genes. Proc Natl Acad Sci USA. 2000;97(17):9597-602. doi:10.1073/pnas.150241797.

10. Mitani K, Kanda Y, Ogawa S, Tanaka T, Inazawa J, Yazaki Y, et al. Cloning of several species of MLL/MEN chimeric CDNAs in myeloid leukemia with $\mathrm{t}(11 ; 19)(q 23 ; p 13.1)$ translocation. Blood. 1995;85(8):2017-24.

11. Rubnitz JE, Behm FG, Curcio-Brint AM, Pinheiro RP, Carroll AJ, Raimondi SC, et al. Molecular analysis of $\mathrm{t}(11 ; 19)$ breakpoints in childhood acute leukemias. Blood. 1996;87(11):4804-8.

12. Takeuchi M, Nakaseko C, Miyagi S, Takeda Y, Ozawa S, Ohwada C, et al. Clonal expansion of non-leukemic cells expressing two novel MLL-ELL variants differing in transforming activity. Leukemia. 2008;22(4):861-4. doi:10.1038/sj.leu.2404954.

13. Yamamoto $K$, Nagata $K$, Tsurukubo $Y$, Inagaki $K$, Ono R, Taki T, et al. Translocation $(8 ; 12)(q 13 ; p 13)$ during disease progression in acute myelomonocytic leukemia with $\mathrm{t}(11 ; 19)(\mathrm{q} 23 ; \mathrm{p} 13.1)$. Cancer Genet Cytogenet. 2002;137(1):64-7.

14. Nilson I, Lochner K, Siegler G, Greil J, Beck JD, Fey GH, et al. Exon/intron structure of the human ALL-1 (MLL) gene involved in translocations to chromosomal region 11q23 and acute leukaemias. Br J Haematol. 1996;93(4):966-72.

15. Kakihana K, Kubo F, Wakabayashi S, Kurosu T, Miki T, Murakami N, et al. A novel variant form of MLL-ELL fusion transcript with $\mathrm{t}(11 ; 19)(\mathrm{q} 23 ; \mathrm{p} 13.1)$ in chronic myelomonocytic leukemia transforming to acute myeloid leukemia. Cancer Genet Cytogenet. 2008;184(2):109-12. doi:10.1016/j. cancergencyto.2008.04.001.

16. De Braekeleer E, Meyer C, Douet-Guilbert N, Morel F, Le Bris MJ, Marschalek R, et al. A complex 1;19;11 translocation involving the MLL gene in a patient with congenital acute monoblastic leukemia identified by molecular and cytogenetic techniques. Ann Hematol. 2009;88(8):795-7. doi:10.1007/s00277-008-0656-8.

17. Lavau C, Luo RT, Du C, Thirman MJ. Retrovirus-mediated gene transfer of MLL-ELL transforms primary myeloid progenitors and causes acute 
myeloid leukemias in mice. Proc Natl Acad Sci USA. 2000;97(20):10984-9. doi:10.1073/pnas.190167297.

18. Burmeister T, Meyer C, Groger D, Hofmann J, Marschalek R. Evidencebased RT-PCR methods for the detection of the 8 most common MLL aberrations in acute leukemias. Leuk Res. 2015;39(2):242-7. doi:10.1016/j. leukres.2014.11.017.

19. Zwaan CM, Kolb EA, Reinhardt D, Abrahamsson J, Adachi S, Aplenc R, et al. Collaborative efforts driving progress in pediatric acute myeloid leukemia. J Clin Oncol. 2015;33(27):2949-62. doi:10.1200/JCO.2015.62.8289.

20. Beach DF, Barnoski BL, Aviv H, Patel V, Schwarting R, Strair R, et al. Duplication of chromosome 1 dup(1)(q21q32) as the sole cytogenetic abnormality in a patient previously treated for AML. Cancer Genetics. 2012;205(12):665-8. doi:10.1016/j.cancergen.2012.09.004.

21. Farina M, Rossi G, Bellotti D, Marchina E, Gale RP. Is having clonal cytogenetic abnormalities the same as having leukaemia? Acta Haematol. 2016;135(1):39-42. doi:10.1159/000437202.

22. Raynaud SD, Brunet B, Chischportich M, Bayle J, Gratecos N, Pesce A, et al. Recurrent cytogenetic abnormalities observed in complete remission of acute myeloid leukemia do not necessarily mark preleukemic cells. Leukemia. 1994;8(2):245-9.

23. Sandoval C, Mayer SP, Giamelli J, Farley T, Ozkaynak MF, Tugal O, et al. Cytogenetic abnormalities during clinical, immunophenotypic, and molecular remission in pediatric acute lymphoblastic leukemia. Cancer Genet Cytogenet. 2000;118(1):9-13.

24. Panagopoulos I, Micci F, Thorsen J, Haugom L, Buechner J, Kerndrup G et al. Fusion of ZMYND8 and RELA genes in acute erythroid leukemia. PLoS One. 2013;8(5):e63663. doi:10.1371/journal.pone.0063663.

25. Panagopoulos I, Torkildsen S, Gorunova L, Tierens A, Tjonnfjord GE, Heim S. Comparison between karyotyping-FISH-reverse transcription PCR and RNA-sequencing-fusion gene identification programs in the detection of KAT6A-CREBBP in acute myeloid leukemia. PLoS One. 2014;9(5):e96570. doi:10.1371/journal.pone.0096570.
26. Panagopoulos I, Gorunova L, Zeller B, Tierens A, Heim S. Cryptic FUS-ERG fusion identified by RNA-sequencing in childhood acute myeloid leukemia. Oncol Rep. 2013;30(6):2587-92. doi:10.3892/or.2013.2751.

27. Nicorici D, Satalan H, Edgren H, Kangaspeska S, Murumagi A, Kallioniemi $\mathrm{O}$, et al. FusionCatcher-a tool for finding somatic fusion genes in pairedend RNA-sequencing data. bioRxiv. 2014. doi:10.1101/011650.

28. Panagopoulos I, Gorunova L, Bjerkehagen B, Heim S. The "grep" command but not FusionMap, FusionFinder or ChimeraScan captures the CIC-DUX4 fusion gene from whole transcriptome sequencing data on a small round cell tumor with $\mathrm{t}(4 ; 19)(\mathrm{q} 35 ; \mathrm{q} 13)$. PLoS One. 2014;9(6):e99439. doi:10.1371/ journal.pone.0099439.

29. Yamamoto K, Seto M, lida S, Komatsu H, Kamada N, Kojima S, et al. A reverse transcriptase-polymerase chain reaction detects heterogeneous chimeric mRNAs in leukemias with 11q23 abnormalities. Blood. 1994;83(10):2912-21.

30. Andersson A, Hoglund M, Johansson B, Lassen C, Billstrom R, Garwicz S, et al. Paired multiplex reverse-transcriptase polymerase chain reaction (PMRT-PCR) analysis as a rapid and accurate diagnostic tool for the detection of MLL fusion genes in hematologic malignancies. Leukemia. 2001;15(8):1293-300.

31. Muntean AG. Mechanisms of mixed-lineage leukemia. Int J Hematol Oncol. 2013;2(3):207-17. doi:10.2217/ijh.13.20.

32. De Braekeleer E, Douet-Guilbert N, Meyer C, Morel F, Marschalek R, De Braekeleer M. MLL-ELL fusion gene in two infants with acute monoblastic leukemia and myeloid sarcoma. Leuk Lymphoma. 2012;53(6):1222-4. doi: 10.3109/10428194.2011.648632.

33. Tuborgh A, Meyer C, Marschalek R, Preiss B, Hasle H, Kjeldsen E. Complex three-way translocation involving MLL, ELL, RREB1, and CMAHP genes in an infant with acute myeloid leukemia and t(6;19;11)(p22.2;p13.1;q23.3). Cytogenet Genome Res. 2013;141(1):7-15. doi:10.1159/000351224.

\section{Submit your next manuscript to BioMed Central and we will help you at every step:}

- We accept pre-submission inquiries

- Our selector tool helps you to find the most relevant journal

- We provide round the clock customer support

- Convenient online submission

- Thorough peer review

- Inclusion in PubMed and all major indexing services

- Maximum visibility for your research

Submit your manuscript at www.biomedcentral.com/submit

\section{() Biomed Central}

